\title{
Isotonies on ordered cones through the concept of a decreasing scale
}

\author{
Gianni Bosi ${ }^{a}$, María J. Campión ${ }^{\mathrm{b}}$, Juan C. Candeal ${ }^{\mathrm{c}}$, \\ Esteban Induráin $^{\mathrm{b}, *}$, Magalì E. Zuanon ${ }^{\mathrm{d}}$ \\ a Dipartimento di Matematica Applicata "Bruno de Finetti”. Università di Trieste. \\ Piazzale Europa 1. I-34127. Trieste, Italy \\ b Departamento de Matemáticas, Universidad Pública de Navarra, Campus Arrosadía, E-31006 Pamplona, Spain \\ c Departamento de Análisis Económico, Facultad de Ciencias Económicas y Empresariales, \\ Universidad de Zaragoza. c/ Doctor Cerrada 1-3, E-50005 Zaragoza, Spain \\ d Dipartimento di Metodi Quantitativi, Università degli Studi di Brescia, Contrada Santa Chiara 50, 25122 Brescia, Italy
}

Received 12 January 2006; received in revised form 10 May 2007; accepted 10 May 2007 Available online 18 May 2007

\begin{abstract}
Using techniques based on decreasing scales, necessary and sufficient conditions are presented for the existence of a continuous and homogeneous of degree one real-valued function representing a (not necessarily complete) preorder defined on a cone of a real vector space. Applications to measure theory and expected utility are given as consequences.

(C) 2007 Elsevier B.V. All rights reserved.

Keywords: Real-valued functions; Numerical representability of ordered structures; Ordered cones; Isotonies; Decreasing scales JEL classification: Primary: C65; Secondary: C00; C60; D11
\end{abstract}

\section{Introduction}

The main purpose of this paper is that of providing a characterization of the existence of a continuous and order-preserving real-valued function defined on a topological preordered cone that also preserves the cone operation (i.e., it is homogeneous of degree one on the strictly positive real numbers).

\footnotetext{
*Corresponding author. Tel.: +34 948 169551; fax: +34 948166057.

E-mail addresses: giannibo@econ.univ.trieste.it (G. Bosi), mjesus.campion@unavarra.es (M.J. Campión), candeal@unizar.es (J.C. Candeal), steiner@unavarra.es (E. Induráin), zuanon@eco.unibs.it (M.E. Zuanon). 
The approach followed to obtain this characterization is based on the existence of particular scales that behave well with respect to the cone operation and that we call homogeneous scales.

The notion of a scale can be understood as a generalization of the Urysohn approach (see Uryshon, 1925) to get continuous functions on a topological space.

This fruitful idea was already used by Nachbin (see Nachbin, 1965) to provide results about the existence of continuous order-preserving functions on preordered topological spaces (see also Burgess and Fitzpatrick, 1977); Herden (1989a).

We go further by extending the previous approach to the algebraical context, a work initiated in Bosi and Zuanon (2003) where the representability of non-complete preorders by means of sublinear utility functionals was analyzed, and in which scales are first used in order to obtain a representation of this kind. Also, for the particular case of semigroups, a pioneer work in this algebraical setting is Bosi et al. (2005).

Although in the present paper we mainly focus on topological preordered real cones, some links with the representability problem of totally preordered topological vector spaces and semigroups (see e.g. Bosi and Zuanon, 2003; Bosi et al., 2005; Candeal et al., 1999; Candeal et al., 2002; Fuchs, 1963; De Miguel et al., 1996; Hofmann and Lawson, 1996) are also considered. This issue is of particular importance in mathematical economics related to constant returns to scale economies (see, e.g., Katzner, 1970, Ch. 2). It should be also noted that all the existing literature concerning this representability problem deals with totally preordered topological real cones. Here, we drop the assumption of the preorder being complete.

As some consequences derived from our main theorem we offer, on the one hand, an application to measure theory that leans on a theorem by Yosida and Hewitt about linear functionals given by a probability measure. On the other hand, we discuss how an expected utility result could be provided, by using the techniques of decreasing scales, whenever the space of lotteries is a totally preordered topological vector space.

\section{Notation and preliminaries}

A preorder $\lesssim$ on a nonempty set $X$ is a reflexive and transitive binary relation on $X$. If, in addition, $\lesssim$ is antisymmetric, then it is said to be an order.

The asymmetric part $\prec$ of a preorder $\lesssim$ is defined as $x \prec y \Longleftrightarrow(x \precsim y) \wedge(\neg(y \precsim x))(x, y \in X)$ and the symmetric part $\sim$ is defined by $x \sim y \Longleftrightarrow(x \precsim y) \wedge(y \precsim x)(x, y \in X)$. A preorder $\precsim$ on $X$ is said to be complete or total if for any two elements $x, y \in X, x \precsim y$ or $y \precsim x$.

A pair $(X, \precsim)$ consisting of a nonempty set $X$ endowed with a preorder $\precsim$ will be referred to as a preordered set. If in addition $X$ is endowed with a topology $\tau$ then the triple $(X, \tau, \precsim)$ is said to be a topological preordered space. In this case, the preorder $\lesssim$ is said to be $\tau$-continuous if, for every $x \in X$, the sets $L(x)=\{t \in X: t \prec x\}$ and $U(x)=\{y \in X: x \prec y\}$ are both $\tau$-open and the sets $D(x)=$ $\{a \in X: a \precsim x\}$ and $I(x)=\{b \in X: x \precsim b\}$ are both $\tau$-closed. Further, if there is a binary operation + on $X$, then we shall use the notation $(X, \tau, \precsim,+)$ (here the binary operation + may or may not be commutative or associative).

We recall that a topological semigroup $(S, \tau,+)$ is a semigroup (i.e., a nonempty set $S$ together with an associative binary operation + ) endowed with a topology $\tau$ on $S$ such that the function $\Phi$ : $S \times S \rightarrow S$ defined by $\Phi(x, y)=x+y(x, y \in S)$ is continuous with respect to the topology $\tau$ on $S$ and the corresponding product topology $\tau \times \tau$ on $S \times S$.

A topological real vector space $E$ is a vector space over the field $\mathbb{R}$ of real numbers, and endowed with a topology $\tau$ that makes continuous the inner operation $+: E \times E \rightarrow E$ and the external operation of product by scalars $(\cdot \mathbb{R}): \mathbb{R} \times E \rightarrow E$. 
A nonempty subset $K \subseteq E$ of a real vector space $E$ is a cone if $\lambda \in(0,+\infty)$ and $x \in K$ imply $\lambda \cdot x \in K$. If the real vector space $E$ is topological, we shall consider on $K$ the restriction of the topology given on $E$.

A cone $K$ of a real vector space $E$ is said to be additive if it remains stable under the internal binary operation + of the vector space, that is, for every $x, y \in K$ it holds that $x+y \in K$.

If $(X, \precsim)$ is a preordered set, a real-valued function $u$ is said to be order-preserving or strictly isotonic if it satisfies the following two conditions:

(i) $x \precsim y \Rightarrow u(x) \leq u(y)(x, y \in X)$.

(ii) $x \prec y \Rightarrow u(x)<u(y)(x, y \in X)$.

In that case the structure $(X, \precsim)$ is said to be representable.

It is clear that if the preorder on $X$ is total, then a real-valued function $u$ on the preordered set ( $X, \precsim)$ is order-preserving if and only if $x \precsim y \Longleftrightarrow u(x) \leq u(y)(x, y \in X)$. In this case $u$ is called a numerical representation, or else a utility function representing the total preorder $\precsim$ on $X$.

A real-valued function $u$ on a set $X$ endowed with a binary operation + is said to be additive if $u(x+y)=u(x)+u(y)(x, y \in X)$ (here again, we do not ask a priori the binary operation + to be commutative or associative).

In the case in which $K$ is a cone of a real vector space, a real-valued function $u: K \rightarrow \mathbb{R}$ is said to be homogeneous of degree one (see Bosi et al., 2000) if $u(\lambda x)=\lambda u(x)(\lambda \in(0,+\infty), x \in K)$. It is said to be homogeneous on rationals if $u(p x)=p u(x)(p \in \mathbb{Q} \cap(0,+\infty), x \in K)$.

When $K$ is an additive cone of a real vector space, and $u$ is an order-preserving function representing a preorder $\lesssim$ defined on $K$, the function $u$ is said to be linear if it is both additive and homogeneous of degree one.

When $(X, \precsim)$ is a preordered set endowed with some binary operation + it is usual to ask the binary operation to satisfy some additional condition of compatibility with the ordering $\precsim$. In this direction, $\precsim$ is said to be translation-invariant if $x \precsim y \Longleftrightarrow x+z \precsim y+z \Longleftrightarrow z+x \precsim z+y(x, y, z \in X)$.

Also, if $K$ is a cone endowed with a preorder $\precsim$, we say that the preorder is homothetic (or compatible with the cone structure) if $x \precsim y \Longleftrightarrow \lambda x \precsim \lambda y(\lambda \in(0,+\infty), x, y \in K)$. It is said to be increasing if for every $x \in K$ and $\lambda, \mu \in(0,+\infty)$ with $\lambda<\mu$ it holds that $\lambda x \prec \mu x$.

Similarly, the preorder $\precsim$ is said to be homothetic on rationals if $x \precsim y \Longleftrightarrow \lambda x \precsim \lambda y(\lambda \in \mathbb{Q} \cap$ $(0,+\infty), x, y \in K)$, and increasing on rationals if for every $x \in K$ and $\lambda, \mu \in \mathbb{Q} \cap(0,+\infty)$ with $\lambda<\mu$ it holds that $\lambda x \prec \mu x$.

If $X$ is any nonempty set endowed with a binary operation +, and $A$ and $B$ are two nonempty subsets of $X$, then define $A+B=\{a+b: a \in A, b \in B\}$.

Also, if $A$ is a nonempty subset of a real vector space, and $\lambda \in \mathbb{R}$ is a real scalar, set $\lambda A=$ $\{\lambda \cdot a: a \in A\}$.

\section{Scales}

Let $X$ be a nonempty set endowed with a topology $\tau$. Let $T$ be a dense subset of the Euclidean real line $\mathbb{R}$ (respectively: of the set $(0,+\infty) \subset \mathbb{R}$. A family $\mathcal{F}=\left\{X_{t}: t \in T\right\}$ of subsets of $X$ is said to be a scale (respectively: a positive scale) on the topological space $(X, \tau)$ if the following conditions hold:

(i) $X_{t}$ is a $\tau$-open subset of $X$ for every $t \in T$.

(ii) $\bar{X}_{s} \subseteq X_{t}$ for every $s, t \in T$ such that $s<t$, where $\bar{Y}$ stands for the $\tau$-closure of a subset $Y \subseteq X$.

(iii) $\cup_{t \in T} X_{t}=X$ and $\cap_{t \in T} X_{t}=\emptyset$. 
Following Nachbin (1965), given a preordered set $(X, \precsim)$ a subset $A \subseteq X$ is called decreasing if for every $x, z \in X$ it holds that $(z \precsim x) \wedge(x \in A) \Rightarrow z \in A$.

Leaning on the concept of a decreasing set, a powerful tool to deal with utility representations of preordered sets was introduced in 1977 by Burgess and Fitzpatrick (see Burgess and Fitzpatrick (1977). This is the key concept of a decreasing scale.

Throughout the paper we shall use a particular case of decreasing scales. Thus, we say that a family $\mathcal{G}=\left\{G_{r}: r \in \mathbb{Q} \cap(0,+\infty)\right\}$ is a positive countable decreasing scale on a topological preordered space $(X, \tau, \precsim)$ if the following conditions hold:

(i) $G_{r}$ is a decreasing subset of $X$ for every $r \in \mathbb{Q} \cap(0,+\infty)$.

(ii) $\mathcal{G}=\left\{G_{r}: r \in \mathbb{Q} \cap(0,+\infty)\right\}$ is a positive scale on $(X, \tau)$.

In the particular case when $\tau$ is the discrete topology on $X$, then a positive countable decreasing scale $\mathcal{G}=\left\{G_{r}: r \in \mathbb{Q} \cap(0,+\infty)\right\}$ will be referred to as a positive countable decreasing pseudoscale on the preordered set $(X, \precsim)$.

\section{Remark 1.}

(i) Our definition of a countable decreasing scale slightly differs from the definition that appears in Burgess and Fitzpatrick (1977). Indeed, in that pioneer paper, in order to study the satisfaction of separation axioms on certain kinds of partially ordered topological space, the authors defined a scale $\mathcal{G}=\left\{G_{r}: r \in \mathcal{S}\right\}$ on a set $X$ endowed with a topology $\tau$ and a partial order $\precsim$, as a family of $\tau$-open decreasing subsets of $X$ indexed in $\mathcal{S}$ as a dense subset of $[0,1]$ containing 1 , and $G_{1}=X$, and further $\bar{G}_{p} \subseteq G_{q}$ whenever $p<q(p, q \in \mathcal{S})$. With this original definition (but dealing with preorders instead of partial orders) we would get orderpreserving functions with values in $[0,1]$. Also, our definition of a countable decreasing scale may also be interpreted as a particular case of the definition of a linear separable system in Herden's terminology (see Herden, 1989a, b), where a linear separable system is a family $\mathcal{E}$ of open decreasing subsets of $X$ which is linearly ordered by set inclusion and such that there exist sets $E_{1}, E_{2} \in \mathcal{E}$ such that $\bar{E}_{1} \subset E_{2}$ and for all sets $E_{1}, E_{2} \in \mathcal{E}$ such that $\bar{E}_{1} \subset E_{2}$ there exists some set $E_{3} \in \mathcal{E}$ such that $\bar{E}_{1} \subset E_{3} \subset \bar{E}_{3} \subset E_{2}$. In Lemma 2.2 of Herden (1995) it has been proved that from every linear separable system it is possible to extract a countable decreasing scale.

(ii) The use of scales in utility theory features many nuances that should be clarified to explain exactly which is the approach that we will follow in the present paper. Thus, if $X$ is a nonempty set endowed with a topology $\tau$, then the $\operatorname{set} \mathcal{C}(X, \mathbb{R})$ of all continuous real-valued functions $f: X \rightarrow \mathbb{R}$ is of interest. As shown later in Lemma 4, that set is equivalent to the set of scales defined on $X$. A scale may be directly interpreted as a continuous real-valued map $g \in \mathcal{C}(X, \mathbb{R})$, and conversely, an element $g \in \mathcal{C}(X, \mathbb{R})$ has associated a scale. If we are looking for continuous maps of the $\operatorname{set} \mathcal{C}(X, \mathbb{R})$ that satisfy additional properties, i.e., if we are interested on a particular subset $\mathcal{S}$ of the $\operatorname{set} \mathcal{C}(X, \mathbb{R})$, it seems natural to explore and characterize the corresponding subset of scales that appears associated to $\mathcal{S}$. To put an example, Lemma 6 in the next section shows that if $X$ is endowed with a preorder $\lesssim$ then the subset $\mathcal{S} \subset \mathcal{C}(X, \mathbb{R})$ of real-valued maps that preserve $\precsim$ and take values on $(0,+\infty)$ is equivalent to the subset of separating positive countable decreasing scales on $(X, \tau, \precsim)$. Focusing in the continuous representability of preordered sets with additional properties of algebraical stuff, in the present paper we characterize the family of scales that is equivalent 
to the subset $\mathcal{T} \subset \mathcal{C}(X, \mathbb{R})$ of continuous order-preserving real-valued maps that are homogeneous of degree one on a structure of a real cone (in other words, they also preserve the cone structure). As far as we now, this algebraical setting has recently been introduced in the literature in papers as Bosi and Zuanon (2003) and Bosi et al. (2005).

(iii) It is well known in utility theory that if $X$ is a nonempty set, and $\lesssim$ is a complete preorder on $X$, then $X$ is representable if and only if there exists a countable subset $\mathcal{F}$ of increasing functions $f: X \rightarrow \mathbb{R}$ such that for every pair $(x, y) \in X \times X$ with $x \prec y$ there exists some function $f \in \mathcal{F}$ such that $f(x)<f(y)$.

This is besides the Debreu approach, a crucial idea behind utility representation theorems for preordered sets. Of course, this central idea can be put in relation to pseudoscales, so that the existence of a countable family of pseudoscales of a certain type would characterize the representability of $(X, \precsim)$ (see e.g. Herden, 1989a,b for more details).

When continuity is also involved, one could explore whether or not the existence of a countable family of scales of a certain type would characterize the continuous representability of $(X, \precsim)$ (see Herden and Pallack, 2002).

Nevertheless, among the different alternative and complementary ways to deal with the concept of a scale, this is not the path we have chosen in the present paper.

Observe that that choice would force us to deal with a countable family of scales to characterize the continuous representability of a given preordered set. As mentioned before, here we will follow a path in which we will characterize that representability by means of only one scale (of a certain type). Then, we will extend that kind of results to the algebraical context of cones, finding scales (but only one in each case!) that characterize the continuous representability of preordered algebraical structures (e.g., semigroups, cones) through order-preserving maps that are algebraical homomorphisms as well.

A positive countable decreasing scale $\mathcal{G}=\left\{G_{r} \quad: \quad r \in \mathbb{Q} \cap(0,+\infty)\right\}$ on a topological preordered space $(X, \tau, \precsim)$ is said to be separating if for every $x, y \in X$ with $x \prec y$, there exist $r_{1}, r_{2} \in \mathbb{Q} \cap(0,+\infty)$ such that $r_{1}<r_{2}$ and $x \in G_{r_{1}} y \notin G_{r_{2}}$.

Moreover, if $X$ is endowed with a binary operation,$+ \mathcal{G}$ is said to be additive if it satisfies the following two conditions:

1. $-G_{q}+G_{r} \subseteq G_{q+r} \quad(q, r, \in \mathbb{Q} \cap(0,+\infty))$.

2. $-\left(X \backslash G_{q}\right)+\left(X \backslash G_{r}\right) \subseteq\left(X \backslash G_{q+r}\right) \quad(q, r, \in \mathbb{Q} \cap(0,+\infty))$.

If $K$ is a real cone, a positive countable decreasing scale $\mathcal{G}$ defined on $K$ is said to be homogeneous if it satisfies the following two conditions:

1. $-q G_{p} \subseteq G_{q p} \quad(p, q, \in \mathbb{Q} \cap(0,+\infty))$.

2. $-q \cdot\left(X \backslash G_{p}\right) \subseteq\left(X \backslash G_{q p}\right) \quad(p, q \in \mathbb{Q} \cap(0,+\infty))$.

We now provide two simple results that establish some natural relations among the concepts introduced above.

Lemma 2. Let $K$ be a real cone endowed with a preorder $\precsim$, and let $\mathcal{G}=\left\{G_{p} \quad: p \in \mathbb{Q} \cap(0,+\infty)\right\}$ be a positive countable decreasing pseudoscale defined on $K$. 
Then the following conditions are equivalent:

(i) $\mathcal{G}$ is homogeneous.

(ii) $q G_{p} \subseteq G_{q p}$ for every $p, q \in \mathbb{Q} \cap(0,+\infty)$.

(iii) $G_{q p} \subseteq q G_{p}$ for every $p, q \in \mathbb{Q} \cap(0,+\infty)$.

(iv) $G_{q p} \subseteq q G_{p}$ for every $p, q \in \mathbb{Q} \cap(0,+\infty)$.

\section{Proof.}

(i) $\Rightarrow$ (ii):

This is obvious.

(ii) $\Rightarrow$ (iii):

Given $x \in G_{q p}$, let us consider $y=\frac{1}{q} x$. It is clear that $y \in \frac{1}{q} \cdot G_{q p}$. By hypothesis $\frac{1}{q} \cdot G_{q p} \subseteq G_{\frac{1}{q} q p}=$ $G_{p} \Rightarrow y \in G_{p}$. Therefore $x=q y \in q G_{p}$.

(iii) $\Rightarrow$ (iv):

This is immediate.

(iv) $\Rightarrow$ (i):

To prove that $q \cdot\left(X \backslash G_{p}\right) \subseteq\left(X \backslash G_{q p}\right) \quad(p, q \in \mathbb{Q} \cap(0,+\infty))$, let $x \in q \cdot\left(X \backslash G_{p}\right)$. Then there exists $y \notin G_{p}$ such that $x=q y$. It follows that $x \notin G_{q p}$ since otherwise $x \in G_{q p} \Rightarrow y=$ $\frac{1}{q} \cdot x \in \frac{1}{q} G_{q p}$. By hypothesis $G_{q p} \subseteq q G_{p}$. Thus $y \in\left(\frac{1}{q} \cdot q\right) G_{p}=G_{p}$, which is a contradiction. To prove that $q \cdot G_{p} \subseteq G_{q p} \quad(p, q \in \mathbb{Q} \cap(0,+\infty))$, let $x \in q \cdot G_{p}$. Take $z \in G_{p}$ such that $x=q z$. If $x \notin G_{q p}$ it would follow, as just proved, that $z=\frac{1}{q} \cdot x \notin G_{\frac{1}{q}} \cdot q p=G_{p}$. But this is a contradiction. Therefore $x \in G_{q p}$ and we are done.

Lemma 3. Let $K$ be an additive cone of a real vector space $E$. Suppose that $K$ is endowed with a preorder $\lesssim$, and let $\mathcal{G}=\left\{G_{p}: p \in \mathbb{Q} \cap(0,+\infty)\right\}$ be a positive countable decreasing pseudoscale defined on $(K,+)$. Then the following properties hold:

(i) If $\mathcal{G}$ is additive, then it is homogeneous.

(ii) If $\lesssim$ is total and translation-invariant, and $\mathcal{G}$ is homogeneous, then $\mathcal{G}$ is additive.

\section{Proof.}

(i) Let $p, q \in \mathbb{Q} \cap(0,+\infty)\}$. Take $x \in G_{p}$. Let $q=\frac{a}{b}$, where $a, b \in \mathbb{N}$. Set $z=\frac{1}{b} \cdot x$. Since $z+\ldots$ ( $b$ times)... $+z=x \in G_{p}$, by additivity of $\mathcal{G}$ it follows that $z \in G_{1} \cdot p$. Consequently, by additivity again, $q x=a \cdot z \in a \cdot G_{\frac{1}{b} \cdot p} \subseteq G_{a \cdot \frac{1}{b} \cdot p}=G_{q p}$. Therefore, by Lemma 2, $\mathcal{G}$ is a homogeneous positive countable decreasing pseudoscale.

(ii) Let $x \in G_{p}$ and $y \in G_{q}$, where $p, q \in \mathbb{Q} \cap(0,+\infty)$. By homogeneity of $\mathcal{G}$, there exist $z$, $t \in G_{1}$ such that $x=p z$ and $y=q t$. Since $\precsim$ is total we can assume, without loss of generality, that $z \precsim t$. Being $p=\frac{a}{b}$ with $b \in \mathbb{N}$, by translation-invariance of $\lesssim$ it follows that $\frac{1}{b} \cdot z \precsim \frac{1}{b} \cdot t$ (otherwise $\left(\frac{1}{b} \cdot t\right)+\left(\frac{1}{b} \cdot t\right) \prec\left(\frac{1}{b} \cdot z\right)+\left(\frac{1}{b} \cdot t\right) \prec\left(\frac{1}{b} \cdot z\right)+\left(\frac{1}{b} \cdot z\right)$, and similarly $t=\left(\frac{1}{b} \cdot t\right)+\ldots(b$ times $) \ldots+\left(\frac{1}{b} \cdot t\right) \prec\left(\frac{1}{b} \cdot z\right)+\ldots(b$ times $) \ldots+\left(\frac{1}{b} \cdot z\right)=z$, which is a contradiction).Therefore, by translation-invariance again we get that $a \cdot\left(\frac{1}{b} \cdot z\right) \precsim a \cdot\left(\frac{1}{b} \cdot t\right)$. Thus $p z \precsim p t$. Once more by translation-invariance, we have that $x+y=p z+q t \lesssim p t+q t=(p+q)$ $t \in(p+q) \cdot G_{1}$. But by homogeneity of $\mathcal{G}$ we have that $(p+q) \cdot G_{1}=G_{p+q}$. Therefore $x+y \in G_{p+q}$. Now let $x \notin G_{p}$ and $y \notin G_{q}$, where $p, q \in \mathbb{Q} \cap(0,+\infty)$. By homogeneity of $\mathcal{G}$, there exist $u$, 
$v \notin G_{1}$ such that $x=p u$ and $y=q v$. Since $\lesssim$ is total we can assume, without loss of generality, that $u \precsim v$. As above, by translation- invariance of $\lesssim$ it follows that $p u \precsim p v$. Again by translationinvariance, we obtain that $x+y=p u+q v \precsim p v+q v=(p+q) v \in(p+q) \cdot\left(K \backslash G_{1}\right)$. But by homogeneity of $\mathcal{G}$ we have that $(p+q) \cdot\left(K \backslash G_{1}\right)=\left(K \backslash G_{p+q}\right)$. Hence $x+y \notin G_{p+q}$ and the proof is finished.

To conclude this section we furnish the following useful lemma that, roughly speaking, makes a "translation", interpreting scales as continuous real-valued functions, and viceversa. Such concepts become equivalent.

Lemma 4. Let $X$ be a nonempty set endowed with a topology $\tau$.

(i) Given a continuous function $u: X \rightarrow \mathbb{R}$, the family $\mathcal{F}=\left\{u^{-1}(-\infty, q): q \in \mathbb{Q}\right\}$ is a scale on $(X, \tau)$.

(ii) Given a scale $\mathcal{F}=\left\{X_{t} \quad: t \in T\right\}$ defined on $(X, \tau)$, where Tis a dense subset of $\mathbb{R}$, it holds that the map $u: X \rightarrow \mathbb{R}$ defined by $u(x)=\inf \left\{t \in T: x \in X_{t}\right\}(x \in X)$, is a continuous function.

Proof. See Gillman and Jerison (1960), pp. 43-44. See also Kelley 1955, Lemma 3 on Ch. 4, as well as Nachbin (1965). Similar ideas had already been introduced in the seminal work due to Urysohn, 1925).

Remark 5. An obvious modification in the statement and proof of Lemma 4 would furnish the equivalence between the concept of a positive scale and that of a continuous real-valued function taking values in $(0,+\infty)$.

\section{Isotonies on ordered cones through the concept of a decreasing scale}

The concept of a decreasing scale allows us to obtain in a straightforward manner a characterization of the existence of a continuous order-preserving function on a preordered topological space (see also Burgess and Fitzpatrick, 1977; Herden, 1989a,b). This result will be used in the sequel.

Lemma 6. Let $(X, \tau)$ be a nonempty topological space, endowed with a pre-order $\lesssim$ (not necessarily complete). The following conditions are equivalent:

(i) There exists a continuous order-preserving function $u:(X, \tau, \precsim) \rightarrow(0,+\infty)$.

(ii) There exists a separating positive countable decreasing scale $\mathcal{G}=\left\{\begin{array}{l}G_{r} \\ : \quad r \in \mathbb{Q} \cap\end{array}\right.$ $(0,+\infty)\}$ on $(X, \tau, \precsim)$.

Proof. See Theorem 3.1 in Bosi et al. (2005) and Remark 5 above. Alternatively, see Theorem 4.1 in Herden (1989a) that is more general.

Remark 7. It is important to observe now that, in the statement of Lemma 6 , the preorder $\lesssim$ has not a priori been compelled to satisfy any restriction, not even $\tau$-continuity. However, the mere existence of a continuous order-preserving function $u:(X, \tau, \precsim) \rightarrow(0,+\infty)$, or, equivalently, of a separating positive countable decreasing scale $\mathcal{G}=\left\{G_{r} \quad: \quad r \in \mathbb{Q} \cap(0,+\infty)\right\}$ on $(X, \tau, \precsim)$, immediately forces the preorder $\precsim$ to be indeed $\tau$-continuous. In other words, Lemma 6 establishes an equivalence between continuous order-preserving functions and separating positive 
countable decreasing scales, but at this point it says nothing about what preorders admit such representations. This complementary problem of looking for conditions (as, e.g., separability in the sense of Debreu case of complete preorders, see Ch. 3 in Bridges and Mehta, 1995), is a horse of a different colour and, at this very stage, it has nothing to do with Lemma 6.

In what follows $K$ will design a nonempty cone of a real topological vector space $E$, and $\precsim$ will denote a preorder defined on $K$. Following Candeal and Induráin (1995), we shall also assume that, unless otherwise stated, the origin $\overline{0} \in E$ does not belong to $K$. This will allow us to deal with situations, involving continuity of representations, that are much more general than those in which the origin must belong to the cone, as studied in Dow and Werlang (1992) or Section II.2 in Keimel and Roth (1992) (For a complete account about this situation, see Candeal and Induráin, 1995; Bosi et al., 2000). Moreover, we generalize the results stated in all these papers to the case of noncomplete preorders.

The following theorem improves Lemma 6, now working on a real cone, and considering continuous and homogeneous of degree one real-valued order-preserving functions.

Theorem 8. Let $K$ be a nonempty real cone of a topological real vector space $(E, \tau)$, and suppose that $K$ is endowed with a preorder $\lesssim$ (not necessarily complete). Assume also that the origin $\overline{0}$ does not belong to $K$. Then the following conditions are equivalent:

(i) There exists a continuous order-preserving function $u$ on $(K, \precsim)$ that takes values in $(0,+\infty)$ and is homogeneous on rationals.

(ii) There exists a continuous and homogeneous of degree one order-preserving function $u$ on $(K, \precsim)$ that takes values in $(0,+\infty)$.

(iii) There exists a homogeneous separating positive countable decreasing scale $\mathcal{G}=$ $\left\{G_{r} \quad: r \in \mathbb{Q} \cap(0,+\infty)\right\}$ on $(K, \precsim)$.

\section{Proof.}

(i) $\Rightarrow$ (ii):

First let us prove that $\precsim$ must in fact be increasing: Observe that $\precsim$ is already increasing on rationals, because, by hypothesis, $u$ is homogeneous on rationals and strictly positive. Now let $x \in K$ and $\alpha, \beta \in(0,+\infty)$ with $\alpha<\beta$. Take $q, r, s, \in \mathbb{Q}$ such that $\alpha<q<r<s<\beta$. Let us prove that $\alpha x \prec r x \prec \beta x$. Since the set $L(q x)=\{y \in K: y \prec q x\}=\mu^{-1}(0, u(q x))$ is, by continuity of $u$, open as regards the topology on $K$, the binary operation $P:(0,+\infty) \times K \rightarrow K$ given by $P(\lambda, x)=$ $\lambda \cdot x \in K$ is continuous, and for every $p \in \mathbb{Q} \cap(0,+\infty)$ with $p<q$ it happens that $p x \prec q x$ because $\precsim$ is increasing on rationals, we have that there exists an interval $\left(p-\epsilon_{p}, p+\epsilon_{p}\right) \subseteq(0,+\infty)$ such that $\in_{p} \in \mathbb{Q} \cap(0,+\infty)$ and $\gamma x \in L(q x)$ for every $\gamma$ in $\left(p-\epsilon_{p}, p+\epsilon_{p}\right)$.

But, by construction, it is clear that $(0, q)$ is a subset of the closure of

$$
A=\underset{p<q: p \in \mathbb{Q} \cap(0,+\infty)}{U}\left(p-\boldsymbol{\epsilon}_{p}, p+\boldsymbol{\epsilon}_{p}\right) .
$$

Given any element in $\lambda \in A$ we know that $\lambda \cdot x \in L(q x)$. Thus $P(A \times\{x\}) \subseteq L(q x)$. Then, by continuity of $P$ (see e.g. Dugundji, 1966, p.80), $P(\bar{A} \times\{x\}) \subseteq \bar{L}(q x)$. Since $U(q x)=\{z \in K: q x \prec z\}=$ $u^{-1}(u(q x),+\infty)$ is an open set that does not meet $L(q x)$, it is plain that $\bar{L}(q x) \subseteq\{t \in K: t \precsim q x\}$. As a consequence, we finally get that $\alpha x \precsim q x$ because $\alpha \in(0, q)$. Therefore $\alpha x \prec r x$, once again 
because $\precsim$ is increasing on rationals. In a completely analogous way we would also obtain that $r x \prec \beta x$. Therefore $\precsim$ is indeed increasing.

Let us prove now that $u$ is homogeneous of degree one: To this task, consider $x \in K$ and $\lambda \in(0,+$ $\infty)$. We have just proved that $\lesssim$ is increasing, so that for every $q \in \mathbb{Q}$ with $\lambda<q$ it follows that $u$ $(\lambda x)<u(q x)=q u(x)$. Since $\lambda=\inf \{q \in \mathbb{Q} \cap(0,+\infty) \quad: \lambda<q\}$, it is plain that $u(\lambda x) \leq \lambda u(x)$. Suppose now, by contradiction, that $u(\lambda x)<\lambda u(x)$. Let $r, r_{1} \in \mathbb{Q} \cap(0,+\infty)$ be such that $\frac{u(\lambda x)}{\lambda}<r<u(x)$ and $\frac{u(\lambda x)}{r}<r_{1}<\lambda$. We have that $u(\lambda x)<r_{1} \cdot r$. Also since $\lesssim$ is increasing and $r_{1}<\lambda$, it follows that $r_{1} x \prec \hat{\lambda} x$. Therefore $r_{1} \cdot u(x)=u\left(r_{1} x\right)<u(\lambda x)<r_{1} \cdot r$. Since $r_{1}>0$, this implies $u(x)<r$. But this is a contradiction.

(ii) $\Rightarrow$ (iii):

The collection $\mathcal{G}=\left\{G_{q}=u^{-1}(0, q) \quad: q \in \mathbb{Q} \cap(0,+\infty)\right\}$ is actually a separating positive countable decreasing scale by Lemma 6 . To prove that $\mathcal{G}$ is also homogeneous, notice that for any $p \in \mathbb{Q} \cap(0,+\infty)$ with $x \in G_{p}$ it holds that $u(x)<p$. Thus for every $q \in Q \cap$ $(0,+\infty)$ we get $q u(x)<q p$. But $K$ is a cone and $u$ is homogeneous on rationals, so we have $u$ $(q x)=q u(x)<q p$ or equivalently $q x \in G_{q p}$. Hence $q \cdot G_{p} \subseteq G_{q p}(p, q \in \mathbb{Q} \cap(0,+\infty))$.

We finally observe that, by Lemma 2 , this is equivalent to the homogeneity of $\mathcal{G}$.

(iii) $\Rightarrow$ (i):

Define $u(x)=\inf \left\{q \in \mathbb{Q} \cap(0,+\infty): x \in G_{p}\right\}$. It follows again by Lemma 6 that $u$ is a continuous real-valued order-preserving function that takes values in $(0,+\infty)$. Let us see that $u$ is homogeneous on rationals: To this task, let $q \in \mathbb{Q} \cap(0,+\infty)$ and $x \in K$. Given any $p \in \mathbb{Q} \cap(0,+\infty)$ such that $x \in G_{p}$, by homogeneity of $\mathcal{G}$ we have that $q x \in q \cdot G_{p} \subseteq G_{q p}$. Thus, by definition of $u$, it is $u(q x) \leq q u(x)$.

Now suppose, by contradiction, that $u(q x)<q u(x)$. Let $r \in \mathbb{Q} \cap(0,+\infty)$ be such that $\frac{u(q x)}{q}<r<u(x)$. By definition of $u$ it follows that $x \notin G_{r}$, so that by homogeneity of $\mathcal{G}, q x \notin G_{q r}$. Again by definition of $u$ we get $q r \leq u(q x)$. Thus $r \leq \frac{u(q x)}{q}$. But this is a contradiction.

This finishes the proof.

\section{Remark 9.}

(i) Along Theorem 8 the preorder $\precsim$ considered on $K \subseteq E$ is not, a priori, forced to satisfy any restriction. In particular, it may or may not be a natural preorder in the cone structure, as defined, for instance, in Keimel and Roth (1992), p. 10 (Remember that a preorder $\lesssim$ on a cone $K$ is said to be natural if for every $x, y \in K$ it holds that $x \precsim y \Longleftrightarrow x+z=y$ for some $z \in K)$.

(ii) From Lemma 3 it follows that if $\precsim$ is total and translation-invariant, and the cone $K$ is additive, the following condition is also equivalent to the ones given in the statement of Theorem 8:

(iv) There exists an additive separating positive countable decreasing scale $\mathcal{G}=\left\{G_{r}: r \in\right.$ $\mathbb{Q} \cap(0,+\infty)\}$ on $(K, \precsim)$.

Actually, if $\lesssim$ is not translation invariant we still have that the implication (iv) $\Rightarrow$ (i) holds true.

As a remarkable fact, notice that this condition (iv) implies that the utility function $u: K \rightarrow(0,+\infty)$ defined in the usual way as $u(x)=\inf \left\{p \in \mathbb{Q} \cap(0,+\infty) \quad: x \in G_{p}\right\} \quad(x \in K)$ is continuous and additive. But, by Lemma 3 , the countable decreasing scale $\mathcal{G}$ is also homogeneous, and consequently $u$ is homogenous of degree one, hence linear, by Theorem 8 . In particular, from Lemma 3 and Theorem 8 we obtain the following easy corollary (that could also be proved using a direct straightforward argument): 
Corollary 10. Let $K$ be a nonempty additive real cone of a topological real vector space $(E, \tau)$, and suppose that $K$ is endowed with a total preorder $\lesssim$ that is representable by a continuous utility function $u: K \rightarrow(0,+\infty)$. Then $u$ is additive if and only if $u$ is linear.

\section{Applications}

We conclude with a construction of additive scales on totally ordered topological vector spaces based on the additive representability of ordered semigroups, an application to measure theory, based on a theorem by Yosida and Hewitt (see Yosida and Hewitt, 1952), and, finally, an application to the study of the representability of lotteries over a totally ordered topological vector space, by means of a particular class of real-valued order-preserving functions.

\subsection{A decreasing scale on the positive cone of a real topological vector space}

Observe that the existence of a continuous and linear utility function to represent a translationinvariant total order $\precsim$ defined on a real topological vector space $E$, is equivalent to the existence of a continuous and linear utility function on its positive cone $E^{+}=\{x \in E: x \prec x+x\}$, which is in particular a totally ordered topological semigroup.

By Remark 9 (ii) this is equivalent to the existence of an additive separating positive countable decreasing scale on $E^{+}$.

If we are able to give a construction of a decreasing scale of such kind on $E^{+}$, we would have at hand a continuous and additive (hence linear by Corollary 10) utility function on $E^{+}$, that can be easily extended to the whole $E$.

Let us give now a direct construction of an additive separating positive countable decreasing scale on $E^{+}$:

We recall that if $(S,+, \precsim)$ is a totally ordered semigroup with $\precsim$ translation-invariant, and $S$ is positive (i.e. $x \prec x+x$, for every $x \in S$ ) then the existence of a continuous and additive utility function $u:(S,+, \precsim) \rightarrow(\mathbb{R},+\leq)$, taking values in $(0,+\infty)$ is indeed equivalent to the satisfaction of the super-Archimedean property (i.e. whenever $x, y \in S$ are such that $x \prec y$, there exists $n \in \mathbb{N}, n \geq 1$ such that $(n+1) \cdot x \prec n y$, where $n z$ means $z+\ldots(n-$ times)... $+z(z \in S)$ (see e.g. De Miguel et al., 1996; Candeal et al., 1997).

To construct the required decreasing scale, fix an element $x_{0} \in E^{+}$, and for every $p=\frac{a}{b} \in \mathbb{Q} \cap$ $(0,+\infty)$, where $a, b$ are positive natural numbers, set $G_{p}=\left\{x \in E^{+}: b x \prec a x_{0}\right\}$. It is straightforward to see that $\mathcal{G}=\left\{G_{p}: \quad p \in \mathbb{Q} \cap(0,+\infty)\right\}$ is an additive separating positive countable decreasing scale, that is also homogeneous by Lemma 3.

\subsection{An application to measure theory}

Consider the space $L^{\infty}(\Omega, F, \mu)$ of all bounded $\mu$-measurable real-valued functions on the measurable space $(\Omega, F, \mu)$, where $F$ is a $\sigma$-algebra of subsets of the nonempty set $\Omega$, and $\mu$ is a $\sigma$-finite measure on $F$. Assume that $L^{\infty}(\Omega, F, \mu)$ is endowed with the essential supremum norm topology $\tau_{\|\cdot\| \|}$ (i.e., $\tau_{\|\cdot\|}$ is the topology induced by the norm $\|x\|=\operatorname{esssup}\{|x(\omega)|: \omega \in \Omega\}\left(x \in L^{\infty}(\Omega\right.$, $F, \mu)$ ), where "esssup" stands for the essential supremum) (For standard definitions concerning Measure Theory related to Functional Analysis see e.g. Rudin (1991)).

A preorder $\lesssim$ on $L^{\infty}(\Omega, F, \mu)$ is said to be monotonic if $x \precsim y$ for all functions $x, y \in L^{\infty}(\Omega, F, \mu)$ such that $x(\omega) \leq y(\omega)$ for almost all $\omega \in \Omega$ (i.e.: $\mu$-almost everywhere). We recall that a finitely 
additive probability $p$ on $(\Omega, F)$ is a function from $F$ into $[0,1]$ such that $p(\emptyset)=0, p(\Omega)=1$, and $p$ $(A \cup B)=p(A)+p(B)$ for all sets $A, B \in F$ such that $A \cap B=\emptyset$. Denote by $\mathbb{1}_{A}$ the indicator function of any set $A \in F$ (i.e., $1_{A}(z)=\mathbb{1}$ if $z \in A$, and $\mathbb{1}_{A}(z)=0$ if $z \notin A$ ).

Proposition 11. Let $\lesssim$ be a monotonic translation invariant total preorder on the $\operatorname{space} L^{\infty}(\Omega, F, \mu)$, and assume that $\mathbb{1}_{\emptyset} \prec 1_{\Omega}$. Then the following conditions are equivalent:

(i) There exists a finitely additive probability p on $(\Omega, F)$, absolutely continuous with respect to $\mu$, such that $E_{p}(x):=\int x(\omega) \mathrm{d} p(\omega)\left(x \in L^{\infty}(\Omega, F, \mu)\right)$ is a utility function for $\precsim$.

(ii) There exists an additive separating positive countable decreasing scale $\mathcal{G}=\left\{G_{r}: r \in\right.$ $\mathbb{Q} \cap(0,+\infty)\}$ on the positive cone of $\left(L^{\infty}(\Omega, F, \mu), \tau_{\|\| \|}, \precsim\right)$.

\section{Proof.}

(i) $\Rightarrow$ (ii):

Assume that there exists a finitely additive probability $p$ on $(\Omega, F)$, absolutely continuous with respect to $\mu$, such that $E_{p}(x):=\int x(\omega) \mathrm{d} p(\omega)\left(x \in L^{\infty}(\Omega, F, \mu)\right)$ is a utility function for $\lesssim$. Then it is clear that the map $E_{p}: L^{\infty}(\Omega, F, \mu) \rightarrow \mathbb{R}$ is a $\tau_{\|\cdot\|}$-continuous, homogeneous of degree one and additive real-valued function, and therefore condition (ii) is verified by Theorem 7 (Observe that the collection $\mathcal{G}=\left\{G_{r}=E_{p}^{-1}(0, r) \quad: r \in \mathbb{Q} \cap(0,+\infty)\right\}$ is actually an additive separating positive countable decreasing scale on the positive cone $\left(L^{\infty}(\Omega, F, \mu)\right)^{+}=$ $\left\{x \in L^{\infty}(\Omega, F, \mu): x \prec x+x\right\}$ since $E_{p}$ is an additive functional that represents $\precsim$ ).

(ii) $\Rightarrow$ (i):

Let $\mathcal{G}=\left\{G_{r}: r \in \mathbb{Q} \cap(0,+\infty)\right\}$ be an additive separating positive countable decreasing scale on the positive cone $\left(L^{\infty}(\Omega, F, \mu)\right)^{+}$of $\left(L^{\infty}(\Omega, F, \mu), \tau_{\|\cdot\|}, \precsim\right)$. From Lemma 2 , we have that $\mathcal{G}$ is also homogeneous. Further, from the Proof of Theorem 7, we have that the map $u:\left(L^{\infty}(\Omega\right.$, $F, \mu))^{+} \longrightarrow(0,+\infty)$ defined by $u(x)=\inf \left\{r \in \mathbb{Q} \cap(0,+\infty): x \in G_{r}\right\}$ is continuous, order-preserving and linear. Moreover, we can extend $u$ to the whole space $L^{\infty}(\Omega, F, \mu)$ by declaring that $u(\overline{0}):=0$, where $\overline{0}$ stands for the null element in $L^{\infty}(\Omega, F, \mu), u(z):=0$, for every $z \in L^{\infty}(\Omega, F, \mu)$ such that $z \sim \overline{0}$, and $u(x):=-u(-x)$ whenever $-x \in\left(L^{\infty}(\Omega, F, \mu)\right)^{+}$. It is important to notice that this extension is also continuous, order-preserving and linear on the whole $L^{\infty}(\Omega, F, \mu)$ (For instance, to check that $u$ is a utility function on $L^{\infty}(\Omega, F, \mu)$, observe that, due to the translation-invariance and completeness of $\precsim$, if $x, y \in\left(L^{\infty}(\Omega, F, \mu)\right)^{+}$are elements such that $x \precsim y$, we get $x \precsim y \Longleftrightarrow x(-x)=\overline{0} \precsim y+(-x) \Longleftrightarrow(-y)+\overline{0} \precsim(-y)+(y-x)=-x)$.

Without loss of generality, we may assume that $u\left(\mathbb{1}_{\Omega}\right)=1$. Therefore, from Theorem 2.3 in Yosida and Hewitt (1952), there exists a finite, finitely additive signed measure $\pi$ on $(\Omega, F)$ such that $u(x)=\int x(\omega) \mathrm{d} \pi(\omega)$ for all $x \in L^{\infty}(\Omega, F, \mu)$ (see also Clark, 2000). Since from the proof of the aforementioned theorem it holds that $\pi(A)=u\left(1_{A}\right)$ for all $A \in F, \pi:=p$ is actually a finitely additive probability (observe that $0=\pi(\emptyset)=u\left(\mathbb{1}_{\emptyset}\right) \leq \pi(A)=u\left(\mathbb{1}_{A}\right) \leq \pi(\Omega)=u\left(\mathbb{1}_{\Omega}\right)=1$ for all $A \in F$ by monotonicity of $\precsim)$, absolutely continuous with respect to $\mu$.

This consideration completes the proof.

\subsection{Numerical representability of a lottery space over a totally ordered topological vector space}

Let $X$ be a nonempty set. We define the lottery space $L(X)$ as the set of all probability distributions on the family $\mathcal{P}_{F}(X)$ of all finite subsets of $X$. In the particular case in which $X$ is a real topological vector space, the elements of $L(X)$ can be reinterpreted as elements of $X$, in the 
following way: Suppose, for instance, that $(p, x, y)$ represents the element in $L(X)$ that assigns probability $p \in[0,1]$ to the singleton $\{x\} \subseteq X$, and probability $1-p$ to the singleton $\{y\} \subseteq X$. Then we would identify $(p, x, y)$ to the element $p x+(1-p) y \in X$.

If $X$ is also endowed with a translation invariant total preorder $\precsim$, the existence of a continuous and linear utility function on $X$, is, by previous results, equivalent to the existence of a continuous and additive utility function on the positive cone $X^{+}$.

It is straightforward to see that the existence of such utility functions is also equivalent to the existence of a continuous utility function $u: L(X) \rightarrow \mathbb{R}$, where $L(X)$ is understood of a subset of $X$ so that it inherits the topology $\tau$ and preorder $\precsim$ defined on $X$, satisfying the following additional property: $u(p, x, y)=p u(x)+(1-p) u(y)$ for every $p \in[0,1]$ and $x, y \in X$.

This kind of utility functions on a lottery space $L(X)$ is usually encountered in expected utility contexts (see Fishburn, 1982 for a complete account). Moreover, some relationship between the existence of such utility functions on a lottery space and additive functions on a suitable ordered semigroup associated to it, was studied in Candeal et al. (2002).

In this direction observe that, by Remark 9 (ii), the existence of such particular class of utility functions on $L(X)$ is equivalent to the existence of a continuous and additive scale on the positive cone $X^{+}$, that is in particular a topological semigroup. Finally, notice that by the application 1 given before, we know how to construct such scales.

\section{Acknowledgements}

This work has been supported by the research project MTM 2006-15025 "Espacios Topológicos Ordenados", of the Spanish Ministry of Culture. We want to express our gratitude to the coparticipants in the research project J.R. De Miguel, O.R. García-Catalán, M.C. Leránoz and M. Zudaire (UPNA. Pamplona, Spain) for their comments and helpful discussions on the manuscript.

Thanks are given to the editor and an anonymous referee for their helpful suggestions and comments.

\section{References}

Bosi, G., Zuanon, M.E., 2003. Continuous representability of homothetic preorders by means of sublinear orderpreserving functions. Mathematical Social Sciences 45 (3), 333-341.

Bosi, G., Candeal, J.C., Induráin, E., 2000. Continuous representability of homothetic preferences by means of homogeneous utility functions. Journal of Mathematical Economics 33, 291-298.

Bosi, G., Campión, M.J., Candeal, J.C., Induráin, E., Zuanon, M.E., 2005. Numerical isotonies of preordered semigroups through the concept of a scale. Mathematica Pannonica 16 (1), 65-77.

Bridges, D.S., Mehta, G.B., 1995. Representations of Preference Orderings. Ed. Springer-Verlag, Berlin.

Burgess, D.C.J., Fitzpatrick, M., 1977. On separation axioms for certain types of ordered topological space. Mathematical Proceedings of the Cambridge Philosophical Society 82, 59-65.

Candeal, J.C., De Miguel, J.R., Induráin, E., 1997. Topological additively representable semigroups. Journal of Mathematical Analysis and Applications 210, 375-389.

Candeal, J.C., Induráin, E., 1995. Homothetic and weakly homothetic preferences. Journal of Mathematical Economics 24, $147-158$.

Candeal, J.C., Induráin, E., Mehta, G.B., 1999. Order preserving functions on ordered topological vector spaces. Bulletin of the Australian Mathematical Society 60, 55-65.

Candeal, J.C., De Miguel, J.R., Induráin, E., 2002. Expected utility from additive utility on semigroups. Theory and Decision 53, 87-94.

Clark, S.A., 2000. The measurement of qualitative probability. Journal of Mathematical Psychology 44, 464-479.

De Miguel, J.R., Candeal, J.C., Induráin, E., 1996. Archimedeaness and additive utility on totally ordered semigroups. Semigroup Forum 52, 335-347. 
Dow, J., Werlang, S.R. da C., 1992. Homothetic preferences. Journal of Mathematical Economics 21, 389-394.

Dugundji, J., 1966. Topology. Allyn and Bacon, Boston.

Fishburn, P.C., 1982. The Foundations of Expected Utility. D. Reidel. Dordrecht, The Netherlands.

Fuchs, L., 1963. Partially Ordered Algebraic Systems. Pergamon Press, Oxford, U.K.

Gillman, L., Jerison, M., 1960. Rings of Continuous Functions. Springer Verlag, New York.

Herden, G., 1989a. On the existence of utility functions. Mathematical Social Sciences 17, 297-313.

Herden, G., 1989b. On the existence of utility functions II. Mathematical Social Sciences 18, 107-117.

Herden, G., 1995. On some equivalent approaches to mathematical utility theory. Mathematical Social Sciences 29, $19-31$.

Herden, G., Pallack, A., 2002. On the continuous analogue of the Szpilrajn Theorem I. Mathematical Social Sciences 43 , $115-134$.

Hofmann, K.H., Lawson, J.D., 1996. Linearly ordered semigroups: historical origins and A.H. Clifford's influence. In: Hofmann, H., Mislove, W. (Eds.), Semigroup theory and its applications. Cambridge University Press, Cambridge, U.K, pp. 15-39.

Katzner, D.W., 1970. Static Demand Theory. Macmillan, London.

Keimel, K., and Roth, W., 1992. Ordered Cones and Approximation. Lecture Notes in Mathematics 1517. Ed. SpringerVerlag. Berlin.

Kelley, J.L., 1955. General Topology. Van Nostrand, New York.

Nachbin, L., 1965. Topology and Order. Van Nostrand Reinhold, New York.

Rudin, W., 1991. Functional Analysis. Mc Graw-Hill, New York.

Uryshon, P., 1925. Über die Mächtigkeit der zusammenhangenden Mengen. Mathematische Annalen 94, $262-295$.

Yosida, K., Hewitt, E.M., 1952. Finitely additive measures. Transactions of the American Mathematical Society 72 , $46-66$. 